$64^{\text {ème }}$ Congrès de la SFCO, 03023 (2016)

DOI: $10.1051 / \mathrm{sfco} / 20166403023$

(C) Owned by the authors, published by EDP Sciences, 2016

\title{
POSTER
}

\section{L'usage de la décompression de kystes maxillaires : présentation de cas, techniques, indications}

\section{Romanet $\mathrm{I}^{1}$, Lan $\mathrm{R}^{2}$, Ordioni $\mathrm{U}^{3}$, Catherine $\mathrm{JH}^{4}$, Pommel L ${ }^{5}$}

1,2,3,4,5 - Service de chirurgie orale Pavillon Dentaire Marseille

ivanromanet@hotmail.fr

Mots clés : Marsupialisation, kystes maxillaire, tumeurs maxillaires, décompression

Un adulte de 25 ans sans antécédents médicaux consulte pour une tuméfaction jugale droite évoluant depuis 5 mois en relation avec des douleurs dentaires du maxillaire droit.

L'examen clinique révèle des mobilités dentaires des dents 15 à 11 , une volumineuse masse maxillaire droite, des adénopathies cervicales.

L'examen panoramique dentaire révèle une volumineuse image radio claire dans la région maxillaire droite.

L'examen par CBCT confirme cette lésion et précise des limites larges en montrant que les corticales vestibulaire et palatine sont rompues.

Le diagnostic de kyste maxillaire est posé.

L'enucléation kystique en première étant trop délabrante, le choix d'une decompression par mise en place de drains bilatéraux placés en muqueuse vestibulaire a été effectué du fait de l'absence de certitude diagnostique (kyste vrai non éliminé).

À 6 mois, le résultat est intéressant avec une réduction de $50 \%$ du volume kystique, une régénération osseuse visible à l'examen $\mathrm{CBCT}$, l'absence de mobilité dentaire.

Nous mettrons en valeur les résultats notables de ce type de traitement par une brève revue de la littérature puis une présentation de plusieurs cas iconographiés.

Cette technique de décompression ainsi que ses indications seront décrites et illustrées.

\section{Références}

1. Pitak-Arnnop P, C haine A, Oprean N, et al Management of odontogenic keratocysts of the jaws : a ten-year experience with 120 consecutive lesions. J Craniomaxillofac Surg $2010 ; 38: 358-64$.

2. Tolstunov $L$, Treasure $T$. Surgical treatment algorithm for odontogenic keratocyst : combined treatment of odontogenic keratocyst and mandibular defect with marsupialization, enucleation, iliac crest bone.

3. Ninomiya T, Kubota Y, Koji T, e tal. Marsupialization inhibits interleukin-1alpha expression and epithelial cell proliferation in odontogenic keratocysts. J Oral Pathol Med $2002 ; 31: 526-33$. 